\title{
Functional grading of metal foam cores for yield-limited lightweight sandwich beams
}

\author{
Yves Conde, Arnaud Pollien, Andreas Mortensen * \\ Ecole Polytechnique Fédérale de Lausanne (EPFL), Laboratory for Mechanical Metallurgy, Institut des Matériaux, \\ Station 12, CH-1015 Lausanne, Switzerland
}

Received 21 February 2005; received in revised form 23 August 2005; accepted 24 October 2005

Available online 21 November 2005

\begin{abstract}
We show that grading the porosity in a bent metal skin/metal foam core sandwich can generate significant weight savings in yieldlimited design when, and only when, there is a gradient in the applied moment along the sandwich beam.

(C) 2005 Acta Materialia Inc. Published by Elsevier Ltd. All rights reserved.

Keywords: Aluminium; Foams; Sandwich structure; Functionally graded materials (FGM); Bending test
\end{abstract}

\section{Introduction}

Porous material structures encountered in nature are often "functionally graded": the material relative density is non-uniform, being distributed in space so as to optimize the global mechanical performance of the structure. One example is provided by bone, which features regions of dense "cortical" bone different from lower-density "trabecular" bone regions. These two regions, and hence the bone relative density, are distributed in space so as to maximize the mechanical performance of a structure such as a femur [1]. Another example is provided by microcellular plant structures, including wood, bamboo, corn or barley stems. In these, a natural mechanism exists for adjusting the local fibre distribution away from low-stress regions towards those regions that experience the highest mechanical stress, again to optimize the overall mechanical performance of the plant structure [2-7].

A similar approach could be of interest in optimizing the design of porous metal structures. We have recently shown that aluminium foam core sandwich structures featuring

\footnotetext{
* Corresponding author. Tel.: +41 2169329 12; fax: +41 216934664. E-mail address: andreas.mortensen@epfl.ch (A. Mortensen).
}

through-thickness gradients in foam density can be produced with the replication process [8]. Experimental results and analysis showed that functional grading of such structures is of essentially no interest for stiffness optimization, the best structure from a specific modulus perspective being always that which features a uniform foam core of the lowest feasible density. On the other hand, data from that study gave indications that the approach may hold some promise for the production of lightweight structures designed to resist the onset of plastic yielding within the porous core.

This observation motivated the present contribution, in which we examine the theoretical potential of metal foam functional grading from the standpoint of yield resistance, focusing on the oft-examined case study of a metal/metal foam sandwich structure subjected to bending [9-11]. We show, using a simplified analysis based on engineering beam theory, that the approach can indeed yield significant weight savings in practical situations.

\section{Analysis}

Consider a symmetric sandwich beam comprising two outer skins of dense metal, separated by a core of foam made of the same metal, deformed in the linear elastic 


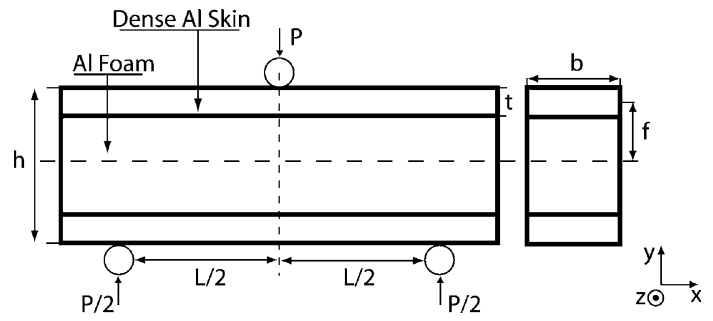

Fig. 1. Foam core sandwich beam in three-point bending, defining geometrical parameters $(f=(h-t) / 2$ is the distance between the centroid of the beam and the centroid of the face).

regime, Fig. 1. The foam pore size is assumed to be sufficiently small in comparison with beam dimensions that the foam material can be treated as an elastoplastic continuum and that strain-gradient effects can be ignored. At length $x$ along the beam axis, the moment is $M$ and the local longitudinal moment gradient is $\mathrm{d} M / \mathrm{d} x$.

In the fully linear elastic regime of deformation, under the assumptions of engineering beam theory the local stress inside the core is characterized by a tensor $\underline{\sigma}$ of the type

$\underline{\sigma}=\left(\begin{array}{ccc}\sigma_{\mathrm{c}} & \tau_{\mathrm{c}} & 0 \\ \tau_{\mathrm{c}} & 0 & 0 \\ 0 & 0 & v \sigma_{\mathrm{c}}\end{array}\right)$

where $\sigma_{\mathrm{c}}$ and $\tau_{\mathrm{c}}$ are the normal and shear stresses in the core along the axis of the beam. The coefficient $v$ allows to adjust the stress in the $z$ direction between plane strain $\left(v=v_{\mathrm{c}}\right.$, the core material Poisson's ratio), and plane stress with $v=0$. As the stress state can be multiaxial in a sandwich core, a multiaxial yield criterion is needed. Elementary engineering beam theory $[12,13]$ dictates that, for elastic deformation

$\sigma_{\mathrm{c}}=E_{(x, y)} y K$

where $y$ is the height across the sandwich beam thickness, $y=0$ corresponding to the neutral axis, $K$ is the beam curvature, and $E_{(x, y)}$ is the foam Young's modulus at point $(x, y) . E_{(x, y)}$ depends on the local volume fraction $V_{\mathrm{f}}$ in the foam core according to

$E_{(x, y)}=C E_{\mathrm{m}}\left(V_{\mathrm{f}}\right)^{2}$

where $C$ is a constant near unity and $E_{\mathrm{m}}$ is the Young's modulus of the constitutive material of the foam [10].

The shear stress $\tau_{\mathrm{c}}$ at location $(x, y)$ inside the core is proportional to the gradient of local moment, $\mathrm{d} M / \mathrm{d} x$, according to

$\tau_{\mathrm{c}}=\frac{\mathrm{d} M}{\mathrm{~d} x}\left(\frac{1}{I b}\right) \int_{y}^{h / 2} E_{(x, y)} y \mathrm{~d} A$

where $I$ is the moment of inertia of the beam, $b$ the width and $A$ the cross-sectional area.

Given the far lower elastic stiffness of metal foams compared with the dense metal they are made from, two approximations can be made [14]: (i) the beam rigidity is entirely governed by uniaxial deformation of the outer skin material. The local beam flexural rigidity $D$ is then given by

$D=2 E_{(x, y)} t b f^{2}$

where dimensions $t, b$ and $f$ are given in Fig. 1. The local stress in the beam core is then a simple function of the local relative density, given the value of the moment $M$ at position $(x, y)$

$\sigma_{\mathrm{c}}=E_{(x, y)} y M D^{-1}$

(ii) the shear stress gradient within the core is negligible. This assumption is legitimate because $\mathrm{d} \tau_{\mathrm{c}} / \mathrm{d} y$ is proportional to the local value of Young's modulus, which is one to two orders of magnitude lower in the core than in the outer skin. As a consequence, the gradient in shear stress is entirely concentrated in the outer skins, such that one can legitimately take $\tau_{\mathrm{c}}$ to be constant across the core

$\tau_{\mathrm{c}}=\frac{\mathrm{d} M}{\mathrm{~d} x} \frac{1}{b f}$

where $f$ is defined in Fig. 1. The precise values of $M$ and $\mathrm{d} M / \mathrm{d} x$, which vary with position $x$ along the beam, depend on the loading configuration of the beam; however, in all events they will be proportional to the applied load $P$

$$
\begin{aligned}
& M=A_{(x)} P \\
& \frac{\mathrm{d} M}{\mathrm{~d} x}=B_{(x)} P
\end{aligned}
$$

In three-point bending, for example, $M$ is given from the point of loading at one end (defined as $x=0$ ) to the central load point at $x=L / 2$ where $L$ is the span length, by the simple expression

$M=\frac{P x}{2}$

such that $\mathrm{d} M / \mathrm{d} x$ is a constant.

Yield within the core will initiate wherever, along the length and across the thickness of the beam, the local stress state meets the yield surface of the core foam material. Using the yield criterion proposed for metal foams by Deshpande and Fleck [15], yielding occurs in the foam when

$\frac{1}{1+\left(\frac{\beta}{3}\right)^{3}}\left[\left(\frac{\sigma_{\mathrm{e}}}{\sigma_{y}}\right)^{2}+\beta^{2}\left(\frac{\sigma_{\mathrm{m}}}{\sigma_{y}}\right)^{2}\right]=1$

where $\sigma_{\mathrm{m}}$ and $\sigma_{\mathrm{e}}$ are the mean and von Mises equivalent stresses respectively

$\sigma_{\mathrm{m}}=\frac{\sigma_{x x}+\sigma_{y y}+\sigma_{z z}}{3}$

$\sigma_{\mathrm{e}}=\frac{1}{\sqrt{2}} \sqrt{\left(\sigma_{x x}-\sigma_{y y}\right)^{2}+\left(\sigma_{y y}-\sigma_{z z}\right)^{2}+\left(\sigma_{z z}-\sigma_{x x}\right)^{2}+6\left(\tau_{x y}^{2}+\tau_{y z}^{2}+\tau_{x z}^{2}\right)}$ 
and $\sigma_{x x}, \sigma_{y y}, \sigma_{z z}, \tau_{x y}, \tau_{y z}, \tau_{x z}$ are the components of the stress tensor. The constant $\beta$ is related to the compressibility of the foam [15]. The denominator is the foam yield stress $\sigma_{y}$, given by [10]

$\sigma_{y}=C^{\prime} \sigma_{y, \mathrm{~m}}\left(V_{\mathrm{f}}\right)^{\frac{3}{2}}$

if we assume that the metal making the foam (and hence the foam) is ideally-plastic. Otherwise, the exponent is $(3+n) /$ 2 , where $n$ is the exponent in the power-law describing the flow curve of the dense metal making the foam [16]. Since $n$ is in practice well below unity, this does not affect the derivation much; hence, we simply take $n=0$ in what follows. The constant $C^{\prime}$ is taken equal to 0.3 , as suggested in [9].

Specializing Eqs. (12) and (13) to the relevant stress state in a metal foam beam core (Eqs. (1), (6) and (7)), we have

$\sigma_{\mathrm{m}}=\sigma_{\mathrm{c}}$

and

$\sigma_{\mathrm{e}}=\frac{1}{\sqrt{2}} \sqrt{2 \sigma_{\mathrm{c}}^{2}+6 \tau_{\mathrm{c}}^{2}}$

Combining Eqs. (8), (9), (11), (15) and (16) one arrives at the local condition that must be obeyed for the core material at position $(x, y)$ not to yield when the applied load is $P$

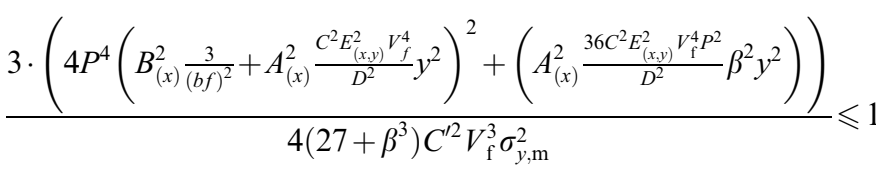

For a beam in three-point bending between $x=0$ and $x=L / 2$, this specializes to

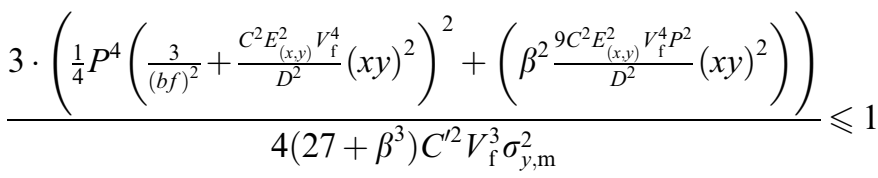

If a sandwich beam of given geometry is to resist load $P$ without failing by local yielding in the core while minimizing the overall core mass, it will be sought to design it such that $V_{\mathrm{f}}$ takes the minimum possible value compatible with Eq. (17) at all points $(x, y)$ within the core. Functional grading of the core may, therefore, be of interest; however, the potential of the approach depends on "real-life" values of material-specific parameters.

\section{Discussion}

The case of pure bending (i.e., the case $B=0$, found for example between the second and third load application points in four-point loading) is of interest in this regard because the ratio of modulus to yield stress (Eqs. (3) and (14)) is a positive power of the foam relative density $V_{\mathrm{f}}$. Therefore, in pure bending it is always beneficial to keep the foam density everywhere as low as possible: the less dense (and hence the weaker but also the more compliant) the foam, the better the beam performance with regard to minimum-weight yield-limited design.

Where $M$ is constant, the core should thus be made with a foam having the lowest relative density possible for the relevant foam production process and resulting foam topology. Obviously, there is no interest in foam grading where $M$ is constant.

When $\mathrm{d} M / \mathrm{d} x$ is finite, on the other hand, the resulting shear stress in the core imposes that a certain minimum foam yield strength, and hence foam volume fraction, be exceeded everywhere within the core. Indeed, as $V_{\mathrm{f}}$ goes to zero, the numerator in Eqs. (17) and (18) no longer tend to zero, whereas the denominator still does: the left-hand side of these equations now diverges to infinity as $V_{\mathrm{f}}$ goes to zero. The minimum local volume fraction that will prevent yielding at load $P$ obviously depends on the beam geometry and the loading situation; however, it now also depends on the coordinates $(x, y)$ of the point under consideration, Eq. (17).

Optimal core design for minimized weight obviously dictates that the foam relative density be exactly at the minimum possible value everywhere, i.e., that it equal that value for which Eq. (17) becomes an equality for the relevant design load $P$. There is hence, where $\mathrm{d} M / \mathrm{d} x$ is finite, an optimal spatial distribution of $V_{\mathrm{f}}(x, y)$ that is non-uniform: grading the core may therefore be of interest. The interesting question is now whether, in practical situations, ensuing gradients in $V_{\mathrm{f}}(x, y)$ are significant, such that grading the foam produces a significant decrease in total beam mass at fixed load $P$. We examine two practical situations in what follows.

We focus on a beam in three-point bending with a distance $L$ of $150 \mathrm{~mm}$ between the external supports and take realistic illustrative values for the different parameters, using Eq. (18) written as an equality to derive a relationship between $y$ and $V_{\mathrm{f}}$. We take $h=20 \mathrm{~mm}$ and $t=$ $1 \mathrm{~mm}$, and consider an open-pore foam for the core, made of $6061 \mathrm{Al}$ alloy in the $\mathrm{T} 6$ condition $\left(\sigma_{y}=275 \mathrm{MPa}\right)$. The foam modulus dependence is taken to be the same as for replicated pure Al, measured in Ref. [16,17]. Thus, we take $C=0.5$.

The result is plotted in Fig. 2 for different values of the design limit applied load $P$, at three positions $x$ along the length of the beam.

As seen, grading the beam is indeed beneficial in weightlimited design, especially when high limit loads are considered (Fig. 2c). The steepest gradient is in each case at $x=75 \mathrm{~mm}$, i.e., where the bending moment is the highest in three-point bending. We can also note that the curves for each value of $x$ converge to a single value of $V_{\mathrm{f}}$ at low $y$ values, i.e., near the neutral axis where shear stresses, constant along $x$, dominate over normal stresses. Fig. 2d) shows the case were yielding has occurred in the beam, at $x=75 \mathrm{~mm}$ (the solution for $V_{\mathrm{f}}$ diverges as $P$ exceeds the beam limit load). 

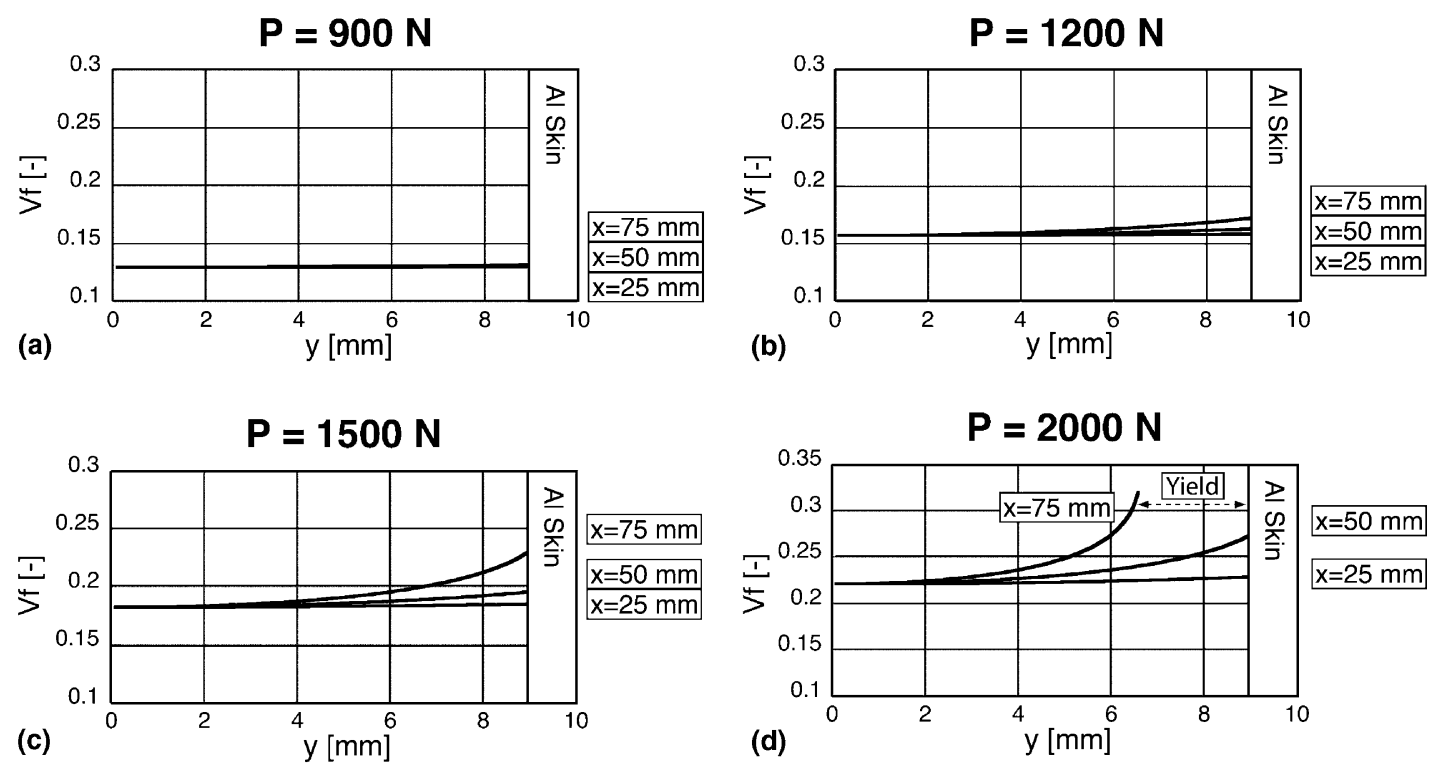

Fig. 2. Optimal $V_{\mathrm{f}}$ distributions for various loads $P$, and at various longitudinal positions $x$, for $h=20 \mathrm{~mm}, t=1 \mathrm{~mm}$ and considering a $6061 \mathrm{Al}$ alloy open-pore foam produced by replication as the core.

A non-graded beam should, for the configuration envisaged here, be made with a $V_{\mathrm{f}}$ of 0.23 to resist a load of $1500 \mathrm{~N}$. With a core of uniform density, the total sandwich beam mass is $49.8 \mathrm{~g}$.

If, instead, the beam is graded, its total mass is given by $m=\rho b \int_{0}^{L}\left(\int_{-h / 2}^{h / 2} V_{\mathrm{f}}(x, y) \mathrm{d} y\right) \mathrm{d} x$

where $S$ is the cross-section surface area of the beam, $L \cdot h$. Eq. (19) solved with $V_{\mathrm{f}}$ given everywhere by Eq. (18) gives a total beam mass $m$ of $43.1 \mathrm{~g}$. Hence, a weight reduction of about $15 \%$ is achieved by grading the beam for this particular case.

As a second example, we calculate the optimal relative density distribution for a beam having a closed-pore Alporas $^{\text {TM }}$ metallic foam core. In this case, Eqs. (20) and (21) are used instead of Eqs. (3) and (14) to describe the Young's modulus and the yield strength of the foam, respectively [10]

$$
\begin{aligned}
& E=C \phi^{2} E_{\mathrm{m}} V_{\mathrm{f}}^{2}+C(1-\phi) V_{\mathrm{f}} \\
& \sigma_{y}=0.3 \phi \sigma_{y, \mathrm{~m}} V_{\mathrm{f}}^{3 / 2}+0.4(1-\phi) V_{\mathrm{f}}
\end{aligned}
$$
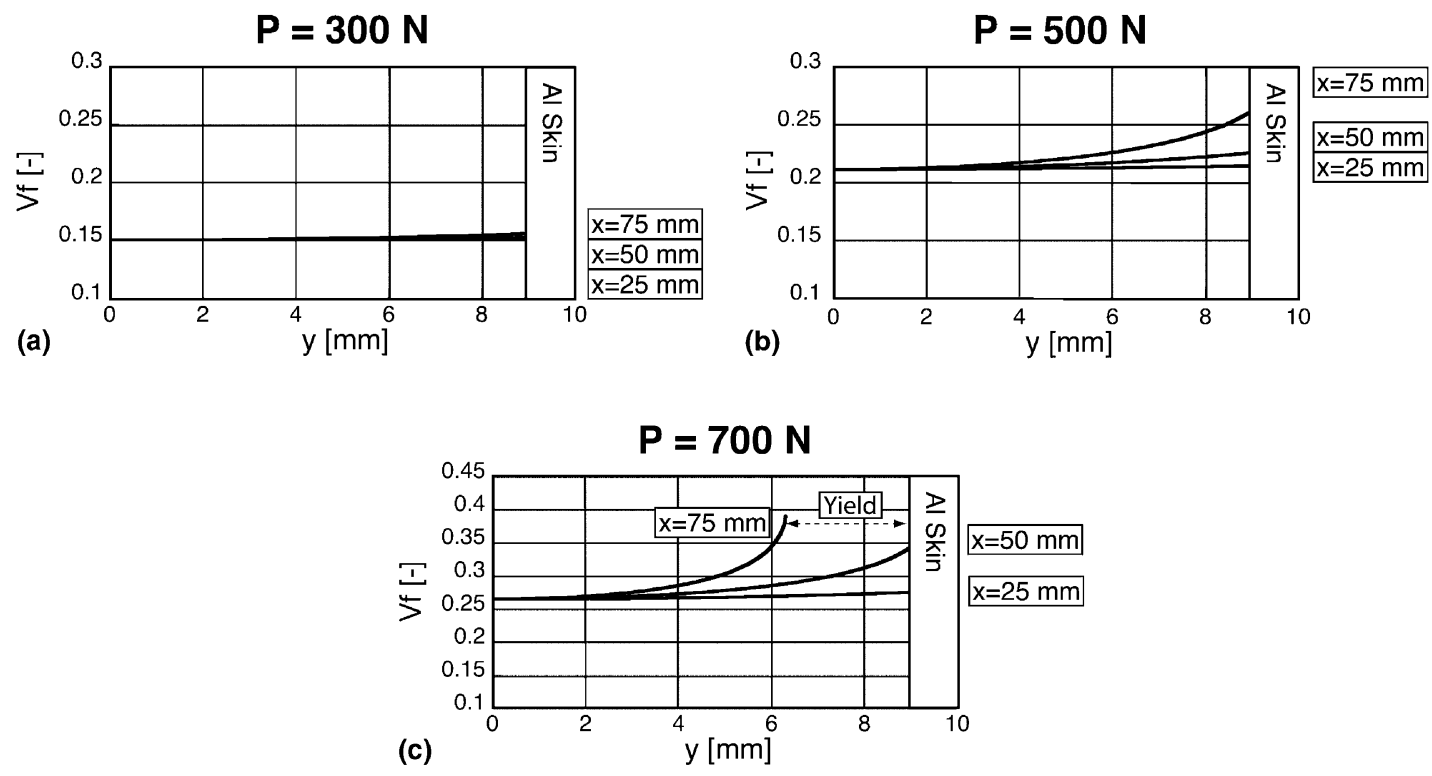

Fig. 3. Optimal $V_{\mathrm{f}}$ distributions for various loads $P$, and at various longitudinal positions $x$, for $h=20 \mathrm{~mm}, t=1 \mathrm{~mm}$ and considering an Alporas ${ }^{\mathrm{m}}$ closed-pore foam for core material. 
We take $C$ as unity and $\phi$, the fraction of solid which is contained in the cell edges, as 0.6 [10]. For the Alporas ${ }^{\mathrm{TM}}$ base material, we take a yield strength $\sigma_{y, \mathrm{~m}}$ of $120 \mathrm{MPa}$ [18]. Results are shown in Fig. 3.

As seen, significant gradients in optimal local porosity exist again, especially at elevated maximum allowable load $P$. Performing an analogous beam mass comparison for the Alporas $^{\mathrm{TM}}$ sandwich beam leads to a weight saving of $15 \%$ when the beam is graded compared to a beam of uniform core density designed to resist the same applied load.

\section{Conclusion}

In conclusion, analysis of a simple metal foam core metal skin sandwich beam in bending shows that, where gradients in applied moment exist along the beam, grading the core density can lead to significant weight savings in yield-limited beam design. For the two specific cases studied, a graded core design provides up to $15 \%$ reduction in total sandwich beam mass at equal maximum allowable load.

The underlying reason for these weight savings is the fact that, where all stress components vary together with the applied load, it will always be best to keep the foam density to its lowest possible value. This is because the modulus (and hence the local stresses) decrease faster with decreasing foam density than does the foam yield stress: when stress components vary everywhere together, lowering the foam density will delay yield.

If, on the other hand, there are within the structure a few regions where stress components do not vary in proportion with one another (as is the case in the core of a sandwich beam wherever the moment varies), designing a structure with a uniform foam density to avoid yield in such regions can lead to significantly overdesigning the foam everywhere else. The global weight penalty paid if the structure is constructed with a uniform foam density may then be high.

\section{Acknowledgement}

This work was supported by the Swiss National Science Foundation (project 200020-100179).

\section{References}

[1] Daxner T, Rammerstorfer FG, Böhm HJ. Mater Sci Technol 2000;16:935.

[2] Nogata F, Takahashi H. Compos Eng B 1995;B5:743.

[3] Nogata F. In: Shiota I, Miyamoto Y, editors. Functionally graded materials 1996. Amsterdam: Elsevier; 1996. p. 737-42.

[4] Amada S. MRS Bull 1995;20:35.

[5] Amada S, Shimizu N. In: Shiota I, Miyamoto Y, editors. Functionally graded materials 1996. Amsterdam: Elsevier; 1996. p. 731-6.

[6] Amada S, Munekata T, Nagase Y, Shimizu N. In: Ilschner B, Cherradi $\mathrm{N}$, editors. Third international symposium on structural and functional gradient materials. Lausanne: Presses Polytechniques et Universitaires Romandes; 1994. p. 689-94.

[7] Parviz N. In: Ilschner B, Cherradi N, editors. Third international symposium on structural and functional gradient materials. Lausanne: Presses Polytechniques et Universitaires Romandes; 1994. p. 695-701.

[8] Pollien A, Conde Y, Pambaguian L, Mortensen A. Graded open-cell aluminum foam core sandwich beams. Mater Sci Eng A 2005;A404:9.

[9] Ashby MF, Evans A, Fleck NA, Gibson LJ, Hutchinson JW, Wadley HNG. Metal foams: a design guide. Boston (MA): ButterworthHeinemann; 2000. 251 pp.

[10] Gibson LJ, Ashby MF. Cellular solids-structure and properties. 2nd ed. Cambridge: Cambridge University Press; 1997. 510 pp.

[11] Evans AG, Hutchinson JW, Ashby MF. Prog Mater Sci 1999;43:171.

[12] Gere JM, Timoshenko SP. Mechanics of materials. Boston (MA): PWS-KENT Publishing; 1990. 807 pp.

[13] den Hartog JP. Strength of materials. New York (NY): Dover Publications; 1949. 323 pp.

[14] Allen HG. Analysis and design of structural sandwich panels. Oxford: Pergamon Press; 1969. 283 pp.

[15] Deshpande VS, Fleck NA. J Mech Phys Solids 2000;48:1253.

[16] San Marchi C, Mortensen A. Acta Mater 2001;49:3959.

[17] Despois JF, Conde Y, San Marchi C, Mortensen A. Adv Eng Mater 2004;6:444.

[18] Andrews E, Sanders W, Gibson LJ. Mater Sci Eng A 1999;A270:113. 